\title{
Einfluss oraler Einnahme von Kollagen-Peptiden auf relevante Parameter der Hautalterung: Hautfeuchtigkeit, Hautelastizität und Hautrauigkeit
}

\author{
Impact of Oral Intake of Collagen-Peptides on Skin Hydration, Elasticity and \\ Roughness
}

Autoren

Institut
G. Schlippe, L. Bolke, W. Voss

Dermatest GmbH, Münster
Bibliografie

Dol http://dx.doi.org/

10.1055/s-0034-1393066

Online-Publikation: 5.10.2015

Akt Dermatol 2015; 41: 529-534

(c) Georg Thieme Verlag KG

Stuttgart · New York

ISSN 0340-2541

\section{Korrespondenzadresse:}

Dr. med. Gerrit Schlippe

Dermatest $\mathrm{GmbH}$

Engelstraße 37

48143 Münster

dr.schlippe@dermatest.de

\section{Zusammenfassung \\ $\nabla$}

Mit zunehmendem Alter und unter Einfluss weiterer Noxen, wie UV-Exposition und Nikotinabusus, kommt es zu einer Abnahme der dermalen extrazellulären Matrix und des Kollagengerüsts. Dies führt zu einer Abnahme der Hautdicke, Elastizität und Feuchtigkeit und damit einhergehend zu einer verstärkten Faltenbildung. Studien ha-

\section{Einleitung \\ $\nabla$}

Das Erscheinungsbild der Haut wird durch intrinsische Faktoren und extrinsische Einflüsse bestimmt. Ziel dieser Einflussfaktoren sind die drei funktionellen Schichten der Haut, die Epidermis, die Dermis mit ihrem Netz aus Kollagen und Elastin und das subkutane Fettgewebe [1].

Zunehmendes Alter und weitere Noxen, wie UVExposition, Nikotinabusus und Ernährungsgewohnheiten, verändern die strukturellen Eigenschaften der Haut und damit das Hautbild. Hierbei kommt es vornehmlich zu einer Abnahme des dermalen Kollagengerüsts, in deren Folge die Hautdicke und Hautfeuchtigkeit abnimmt. Im weiteren Verlauf verringert sich die Elastizität der Haut und Falten entstehen [2].

Kosmetische Interventionen versuchen seit jeher eine gesunde und jugendliche Erscheinung der Haut zu ermöglichen. Hierbei geht es zum einen um präventive - also zustandserhaltende - als auch um therapeutische Maßnahmen, die einen bestimmten Zustand wiederherstellen sollen. Während topische Anwendungen seit langer Zeit etabliert sind, liegt der Fokus in letzter Zeit auf systemischen Anwendungen, die die Haut am ganzen Körper beeinflussen. Da Kollagen eines der häufigsten Strukturproteine im menschlichen Körper ist und als wesentlicher Bestandteil der extrazellulären Matrix der tieferen Hautschichten die Hautdicke, Hautfeuchtigkeit und Elastizität bestimmt, liegt der Fokus im Bereich der systemi- ben gezeigt, dass die orale Aufnahme von speziellen Kollagen-Peptiden über einen längeren Zeitraum die Hautphysiologie positiv beeinflusst. In der vorliegenden klinisch-dermatologischen Prüfung zeigte die Einnahme von kurzkettigen Kollagen-Peptiden (Prüfpräparat: ELASTEN ${ }^{\circledR}$ ) über einen Zeitraum von 3 Monaten signifikant positive und nachhaltige Effekte auf die Hautparameter Elastizität, Feuchtigkeit und Hautrauigkeit.

schen Anwendungen in den letzten Jahren auf der oralen Einnahme spezieller, hydrolysierter Kollagen-Peptide ( $\bullet$ Abb. 1).

Vorangegangene Studien, die die orale Aufnahme von definierten Kollagen-Peptiden untersucht haben, konnten zeigen, dass sich relevante Parameter wie Hautfeuchtigkeit, Hautelastizität und Hautrauigkeit sowie die Hautbarriere signifikant verbessern [3-6].

Die Kollagen-Peptide werden über den gastrointestinalen Trakt aufgenommen, sind anschließend im Blut nachweisbar und akkumulieren in der Haut [7 -9]. Hier führen sie im Vergleich zum Placebo zu einem signifikanten Anstieg von Procollagen Typ 1 um 65\% und einem signifikanten Anstieg von Elastin um 18\% [3].

In der vorliegenden offenen, unkontrollierten, monozentrischen klinisch-dermatologischen Prüfung wurde der Einfluss eines Nahrungsergänzungsmittels mit kurzkettigen Kollagen-Peptiden (Prüfpräparat: ELASTEN ${ }^{\circledR}$ ) auf die physiologischen Hautparameter Elastizität, Feuchtigkeit und Rauigkeit überprüft.

\section{Methoden \\ $\nabla$}

Testpersonen

Untersucht wurden 16 hautgesunde, weibliche Probanden mit trockener Haut im Alter von 45 60 Jahren $(54,25 \pm 4,19$ Jahre). Alle Probanden gaben eine schriftliche Einverständniserklärung 


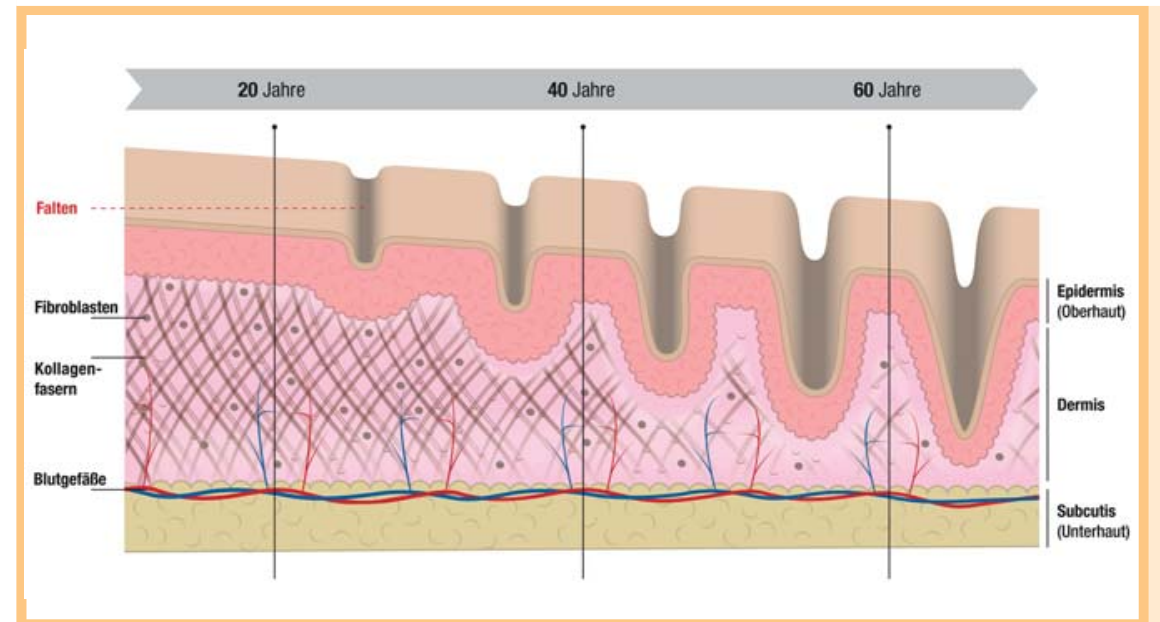

Abb.1 Querschnitt Hautalterung.

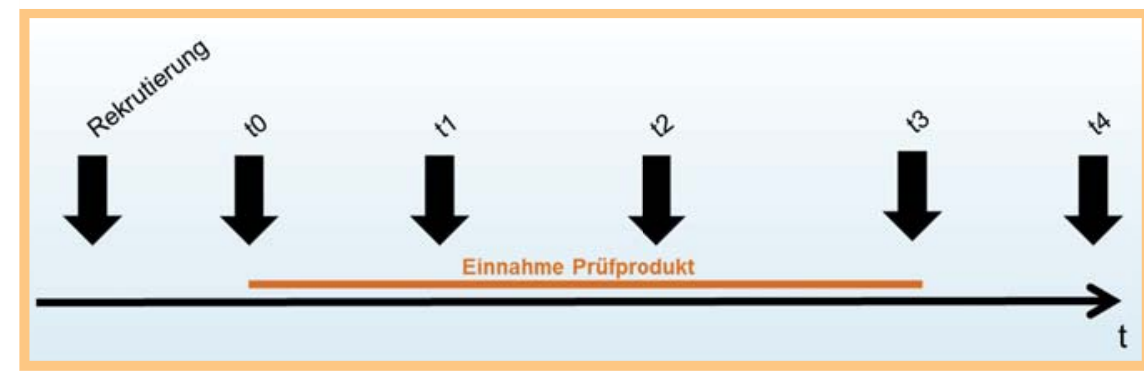

Abb.2 Darstellung der Untersuchungszeitpunkte.

ab und waren in der Lage, mit dem Prüfarzt zu kommunizieren, die Anforderungen dieser klinischen Prüfung zu verstehen und zu befolgen. Die Studie wurde von der Freiburger Ethik-Kommission am 19.5.2014 beratschlagt und unter Zeichen 014/1385 zustimmend bewertet.

Als Ausschlusskriterien galten schwere oder chronische Hautentzündungen, schwere innere oder chronische Erkrankungen, Einnahme von Medikamenten, die die Hautreaktion beeinträchtigen können, Glukokortikoide, Antiallergika, topische Immunmodulatoren, etc., Applikation von wirkstoffhaltigen Präparaten und Pflegemitteln 7-10 Tage vor Testbeginn, schwere Allergien oder jemals aufgetretene schwerwiegende Nebenwirkung durch kosmetische Präparate, Sonnenbäder oder Solariumbesuche während der Studie, bekanntes Krebsleiden, Schwangerschaft und Stillzeit. Die Probanden dürfen im Lauf der klinischen-dermatologischen Untersuchung keine Änderung an ihrem Lebensstil, an eventuellen Genussgewohnheiten (Rauchen, Alkoholkonsum) vornehmen und die Aufnahme oder Aussetzung einer Behandlung mit Östrogenen und Progesteron nicht ändern.

Die Untersuchungen wurden vom Institut Dermatest ${ }^{\circledR}$ in Münster durchgeführt.

\section{Prüfprodukt}

Das Prüfprodukt ELASTEN ${ }^{\circledR}$ enthält folgende Inhaltsstoffe pro Trinkampulle ( $25 \mathrm{ml}$ ): 2,5 g Kollagen-Peptide, $666 \mathrm{mg}$ Acerolafrucht-Extrakt, $80 \mathrm{mg}$ Vitamin C, $3 \mathrm{mg}$ Zink, 2,3 mg Vitamin E (aus natürlichen gemischten Tocopherolen), 50 $\mu \mathrm{g}$ Biotin. Über einen Zeitraum von 12 Wochen nahmen die Probanden eine Trinkampulle $(25 \mathrm{ml})$ pro Tag zu sich.

\section{Untersuchungsbereiche}

Die Messungen der Hautfeuchtigkeit und der Hautelastizität wurden am Unterarm vorgenommen. Die Hautrauigkeit wurde mithilfe von Hautrepliken des lateralen Gesichtsbereichs bestimmt.

\section{Untersuchungszeitpunkte}

Die Untersuchungen fanden vor der ersten Einnahme (t0) nach 4 $(\mathrm{t} 1), 8(\mathrm{t} 2)$ und $12(\mathrm{t} 3)$ Wochen, sowie $4(\mathrm{t} 4)$ Wochen nach der letzten Einnahme statt $(\bullet$ Abb.2).

\section{Messung der Hautfeuchtigkeit}

Die Messung der Hautfeuchtigkeit mittels Kapazitätsmessung wurde mit dem Corneometer ${ }^{\circledR}$ CM 825 (Courage und Khazaka electronic $\mathrm{GmbH}$ ) durchgeführt, welcher die reaktive Kapazität des Stratum corneum misst. Die Probanden wurden 45 Minuten bei einer Temperatur von 22 Grad Celsius und 60\% relativer Feuchtigkeit klimatisiert. Hautmesswerte werden im jeweiligen Testfeld an drei verschiedenen Stellen gemessen. Die gefundenen Werte wurden gemittelt.

\section{Messung der Hautelastizität}

Die Untersuchungen zur Hautelastizität wurden mit dem Cutometer ${ }^{\circledR}$ MPA 580 (Courage und Khazaka electronic GmbH) durchgeführt. Die Bruttoelastizität der Haut wurde nach folgender Formel berechnet: $R 2=(e(a)-e(a+b)) / e(a)=U a / U f$ mit $a=$ Ansaugzeit, $b=$ Relaxationszeit, $e(x)=$ Amplitude an der Stelle $t=x$. Als Geräteeinstellungen wurde der Unterdruck auf 450 mbar eingestellt. Die Ansaugzeit wurde auf 5 Sekunden festgelegt. Die Relaxationszeit (Zeit, in der der Druck abrupt wegfällt) wurde auf 3 Sekunden festgelegt. Insgesamt wurden pro Messung drei Wiederholungen durchgeführt. Pro Test- und Kontrollareal wur- 


\begin{tabular}{|llllll|l|}
\hline & $\mathbf{t 0}$ & $\mathbf{t 1}$ & $\mathbf{t 2}$ & $\mathbf{t 3}$ & $\mathbf{t 4}$ & $\begin{array}{l}\text { Tab. } \mathbf{1} \text { Ergebnisse der Corneo- } \\
\text { metrie-Messung. }\end{array}$ \\
\hline Mittelwert & 35,52 & 40,87 & 42,33 & 43,18 & 41,16 & \\
\hline SEM & 0,846 & 1,220 & 1,144 & 0,932 & 0,926 & \\
\hline p-Wert & & $\leq 0,001$ & 0,021 & 0,065 & $\leq 0,001$ & \\
\hline
\end{tabular}

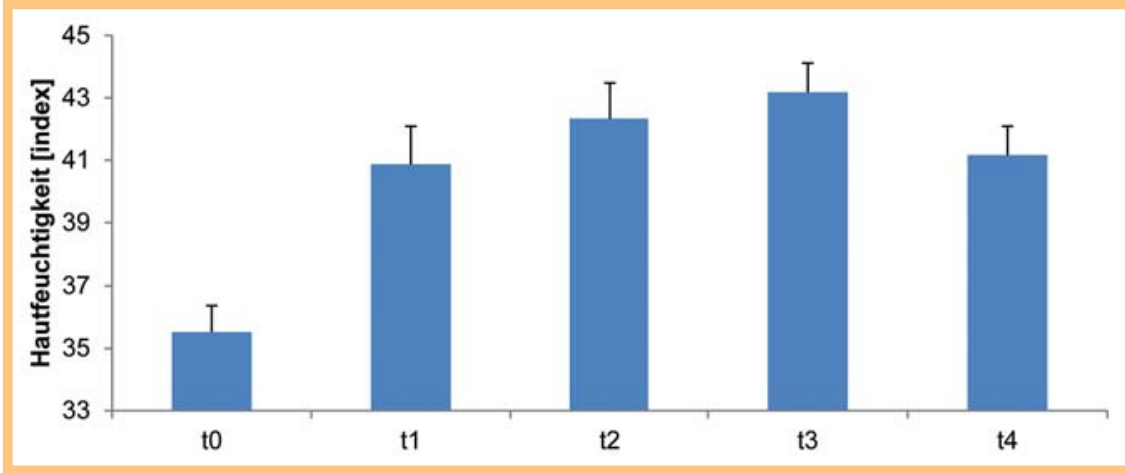

Abb.3 Ergebnisse der Corneometrie-Messung.

den drei Messzyklen durchgeführt und die R2-Werte der drei Messzyklen gemittelt.

\section{Messung der Hautrauigkeit}

Die Messung der Hautrauigkeit wurde mittels optischer 3D-Erfassung und Bewertung der Hautoberfläche mit der PRIMOS-Methode (Phaseshift Rapid In vivo Measurement Of Skin) (GFMesstechnik, Teltow, Germany) durchgeführt. Hierzu wurden Repliken (Hautabdrücke auf Silikonbasis) erstellt, um Messungenauigkeiten durch Körperbewegungen zu vermeiden. Mit der PRIMOS ${ }^{\circledR}$ Software lassen sich die Strukturveränderungen der Oberhaut mithilfe der verschiedenen nach DINund ISO genormten Oberflächenmessgrößen quantitativ zuordnen. Der Rz-Wert (DIN) beschreibt hierbei das arithmetische Mittel aus den Einzelrautiefen fünf aneinander grenzender, gleichlanger Einzelmessstrecken des (digital) gefilterten Profils.

\section{Bildgebung über das VISIA ${ }^{\text {TM-System }}$}

Mit Hilfe des VISIA ${ }^{\mathrm{TM}}$-Systems (Canfield Scientific) wurden digitale Aufnahmen des Gesichtes erstellt. Die Apparatur mit Kinnauflage und Arretierung auf Stirnhöhe ermöglicht die Aufnahme des Hautzustandes unter gleichen Bedingungen wie z. B. Abstand zur Kamera, Belichtung, Perspektive und die genaue Angleichung des Messareals. Hierdurch können „Vorher“- und „Nachher“-Situation optimal verglichen werden. Dazu werden Einzelaufnahmen des Gesichts aus drei verschiedenen Positionen zu den betreffenden Messzeitpunkten aufgenommen und ausgewertet.

\section{Erfassung des subjektiven Eindrucks der Probandinnen}

Zusätzlich zu den oben beschriebenen Parametern wurde der subjektive Eindruck der Probandinnen mittels Fragebogen über den gesamten Studienzeitraum erfasst. In 24 Fragen wurden folgende Bewertungskriterien erfasst: allgemeine Beurteilung des Produktes, subjektives Hautgefühl nach der Anwendung des Produktes, subjektiver Effekt der Produkteinnahme nach definiertem Zeitraum.

\section{Statistik}

Prüfung der Daten auf Normalverteilung mittels KolmogorovSmirnov-Test. Prüfung auf signifikante Unterschiede mittels student'schem t-Test. Ein p-Wert von $<0,05$ galt als statistisch signifikant. Dargestellt sind die Mittelwerte und Standardfehler.

\section{Ergebnisse \\ $\nabla$}

\section{Dermatologische Untersuchungen}

Alle Testpersonen zeigten eine gesunde Haut. Pathologische Hautveränderungen waren in keiner Form festzustellen. Keine der Testpersonen zeigte im Verlauf der Einnahme auffällige unerwünschte oder pathologische Hautveränderungen. Zusätzlich kam es zu keiner Form von anderweitiger Unverträglichkeit oder unerwünschten Reaktionen auf das Produkt. Testunterbrechungen oder gar hautfachärztliche Behandlungen wurden in keinem Fall nötig. Bei der dermatologischen Abschlussuntersuchung nach dem Anwendungsende zeigten sich bei keiner der Testpersonen pathologische Hautveränderungen. Zusätzlich kam es zu keiner Form von Unverträglichkeit oder unerwünschten Reaktionen auf das Produkt. Testunterbrechungen oder gar hautfachärztliche Behandlungen wurden auch nach der Abschlussuntersuchung in keinem Fall nötig.

\section{Hautfeuchtigkeit}

Die Hautfeuchtigkeit nahm nach 4 Wochen und im weiteren Verlauf nach 8 Wochen signifikant zu. Eine weitere Zunahme der Hautfeuchtigkeit nach 12 Wochen zeigte sich im Trend, wobei keine statistische Signifikanz erzielt wurde. Vier Wochen nach Beendigung der Einnahme ging die Hautfeuchtigkeit dann signifikant, aber geringfügig zurück bei immer noch deutlich höheren Werten in Bezug auf to. So lag die Abnahme vier Wochen nach Beendigung der Einnahme bei lediglich 5\% ( $\bullet$ Tab. 1, $\bullet$ Abb.3).

\section{Hautelastizität}

Über einen Zeitraum von 12 Wochen zeigte sich eine signifikante Zunahme der Hautelastizität. Vier Wochen nach der letzten Einnahme nahm die Hautelastizität im Vergleich zu t3 signifikant geringfügig um $5 \% \mathrm{ab}$, sodass im Vergleich zu to immer noch deutlich höhere Elastizitätswerte gemessen wurden ( $\bullet$ Tab.2, (- Abb.4). 


\begin{tabular}{|llllll|l|}
\hline & $\mathbf{t 0}$ & $\mathbf{t 1}$ & $\mathbf{t 2}$ & $\mathbf{t 3}$ & $\mathbf{t 4}$ & $\begin{array}{l}\text { Tab. } 2 \text { Ergebnisse der Cutome- } \\
\text { trie-Messung. }\end{array}$ \\
\hline Mittelwert & 0,607 & 0,669 & 0,708 & 0,729 & 0,691 & 0,01 \\
\hline SEM & 0,01 & 0,01 & 0,01 & 0,01 & $\leq 0,001$ \\
\hline
\end{tabular}

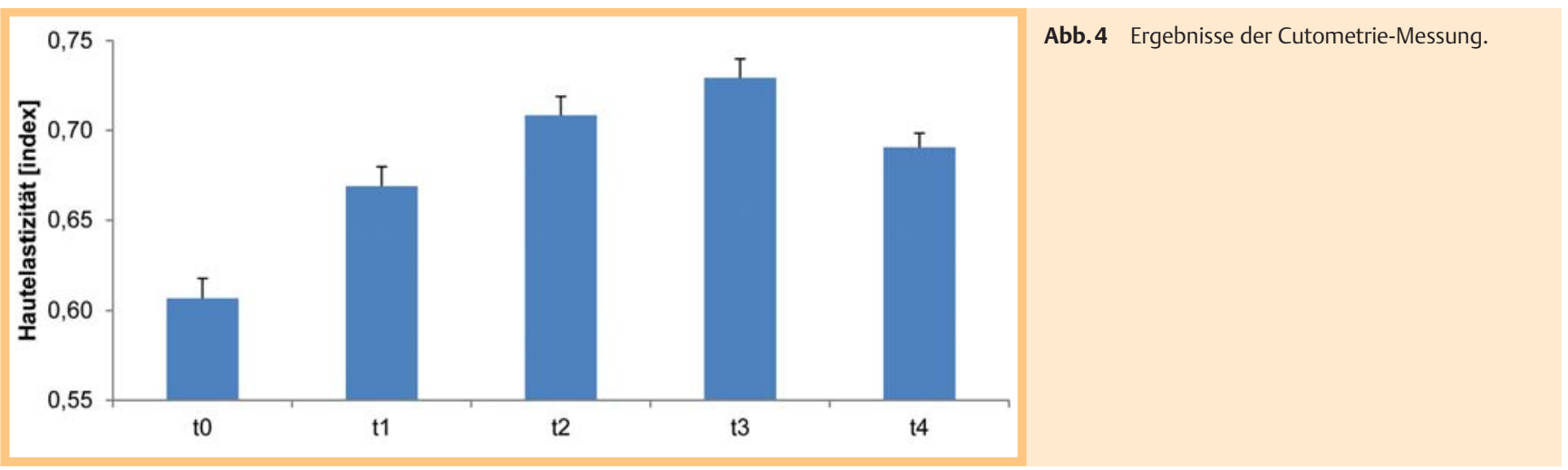

\begin{tabular}{|llllll|} 
& t0 & $\mathbf{t 1}$ & $\mathbf{t 2}$ & $\mathbf{t 3}$ & $\mathbf{t}$ \\
\hline Mittelwert & 156,5 & 141,8 & 133,0 & 130,8 & 135,167 \\
\hline SEM & 3,20 & 5,03 & 4,43 & 4,47 & 3,76 \\
\hline p-Wert & & 0,001 & 0,005 & 0,039 & 0,038 \\
\hline
\end{tabular}

Tab. 3 Ergebnisse der optischen 3D-Messung der Hautoberfläche.

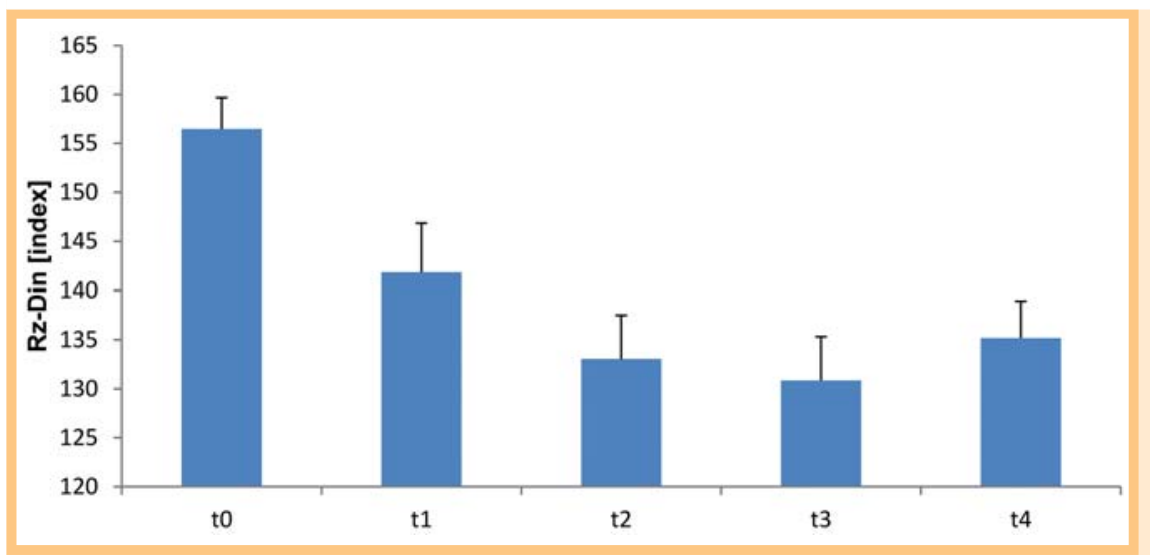

Abb.5 Ergebnisse der optischen 3D-Messung der Hautoberfläche.

\section{Optische 3D-Messung der Hautoberfläche}

Die Ergebnisse der optischen 3D-Messung der Hautoberfläche zeigten signifikante Verbesserungen der Hautrauigkeit bereits nach 4 Wochen mit weiterer signifikanter Abnahme der Hautrauigkeit bis t3. Vier Wochen nach der letzten Einnahme zeigte sich dann wieder eine leichte, signifikante Zunahme der Hautrauigkeit um 3\% (n=6) ( Tab.3, $\bullet$ Abb.5 und $\bullet$ Abb.6).

\section{Diskussion}

Erste Anzeichen der Hautalterung treten schon ab dem 25. Lebensjahr auf. Mit zunehmendem Alter ist die Bildung von Falten die offensichtlichste Form der Hautalterung [2]. Obwohl das Altern als physiologischer Vorgang ein elementarer Bestandteil des Lebens ist, ist der Alterungsprozess von einer großen interindividuellen Heterogenität geprägt. Maßgeblich sind intrinsische und extrinsische Faktoren. Extrinsische Faktoren sind verhaltensabhängig und offenbar für bis zu 80\% der Hautalterung verant- wortlich. Wichtige extrinsische Einflussfaktoren sind UV- und IRStrahlung, Ernährung, Alkohol- und Nikotinkonsum. Die intrinsischen Alterungsprozesse korrelieren i.d.R. mit dem Alter. Hier spielt die genetische Disposition die wichtigste Rolle. Grundsätzlich gilt, dass die intrinsischen Faktoren nicht direkt beeinflussbar sind.

Unabhängig von den intrinsischen und extrinsischen Faktoren nimmt die dermatologische Beeinflussung von Alterserscheinungen - vor allem der sichtbaren Haut - einen immensen Stellenwert ein. Seit einigen Jahren spielen in diesem Segment neben topischen auch systemische Anwendungen - häufig als Nahrungsergänzungsmittel - eine zunehmend wichtige Rolle. Hierbei werden neben vielfältigen und im Wesentlichen aus dem Mineralstoff-, Vitamin- und pflanzlichen Bereich stammenden Substanzen nun auch hydrolysierte Kollagen-Peptide eingesetzt, die in Studien signifikante Effekte auf relevante Parameter der Hautalterung aufwiesen [3-5,10-14]. Zusätzlich zu den Effekten der Hautalterung, der Hautelastizität und der Hautrauigkeit sind positive Effekte auf die Hautbarriere und genetische Faktoren gezeigt worden $[6,14]$. Während die zugrundeliegenden 


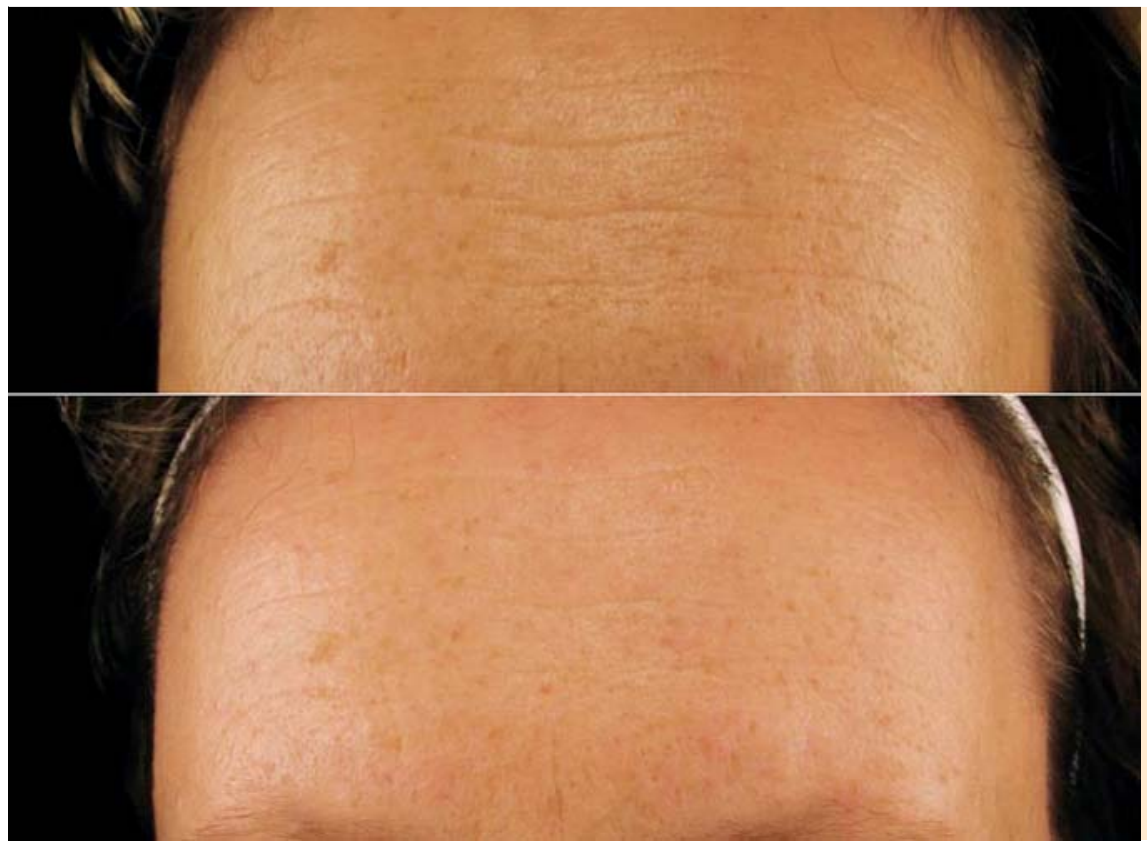

Abb.6 Sichtbare Faltenreduktion im Bereich der Stirn nach 12-wöchiger Einnahme von KollagenPeptiden in den VISIA-Aufnahmen. Oben: vor der Einnahme (t0). Unten: nach 12-wöchiger Einnahme (t3).

Mechanismen noch nicht in Gänze verstanden sind, zeigten Studien, dass Kollagen-Peptide die Proliferation von Fibroblasten und die Synthese von Hyaluronan erhöhen $[15,16]$.

Die systemische Zufuhr von Kollagen-Peptiden, Vitamin C, Biotin und Zink über einen Zeitraum von 3 Monaten führte in dem hier untersuchten Kollektiv zu einer signifikanten Zunahme der Hautfeuchtigkeit um 22\%, während die Hautelastizität um 20\% zunahm. Die Hautrauigkeit ging um 17\% - in Einzelfällen um bis zu 32\% - zurück. Die Ergebnisse der Follow-up-Untersuchungen zeigten, dass es bei allen drei Parametern nach Beendigung der Einnahme zu einer signifikanten, aber nur geringen Abnahme der Effekte zwischen 3 und 5\% kam. Diese Daten verdeutlichen die Nachhaltigkeit der Effekte.

Die systematischen Erfassungen des subjektiven Eindrucks der Probandinnen vor, während und nach der Interventionsphase bestätigen außerdem die positiven Messergebnisse. Dreiviertel der Anwenderinnen fühlten nach der Einnahme eine straffere und feinere Haut. Über $70 \%$ der Anwenderinnen verspürten nach der Anwendung eine verbesserte Hautelastizität und eine bessere Hautfeuchte. $63 \%$ der Anwenderinnen berichteten nach der Anwendung über ein generell frischeres Aussehen.

Die Ergebnisse dieser klinisch-dermatologischen Prüfung zeigen, dass die systemische Einnahme speziell definierter KollagenPeptide in Kombination mit Vitamin C, Zink und Biotin bei sehr guter Verträglichkeit signifikante Effekte auf relevante Hautparameter wie Hautfeuchtigkeit, Hautelastizität und Hautrauigkeit hat.

\section{Interessenkonflikt}

Sponsor der Studie ist die QUIRIS Healthcare GmbH \& Co. KG, Gütersloh.

\section{Abstract}

\section{Impact of Oral Intake of Collagen-Peptides on Skin Hydration, Elasticity and Roughness \\ $\nabla$}

With increasing age, the dermal matrix and collagen content decreases which results in a loss of skin hydration, elasticity and increased wrinkling. In addition to the normal ageing process, several other factors like UV radiation or smoking negatively influence skin physiology. Prior studies demonstrated that the oral intake of bioactive collagen peptides induces beneficial effects on human skin structure and functioning. In this study the oral intake of special collagen peptides (investigational product: ELASTEN $^{\circledR}$ ) for 3 months results in significant and sustainable improvements in skin parameters including skin hydration, elasticity and roughness.

\section{Literatur}

1 Smalls LK, Wickett R, Visscher MO. Effect of dermal thickness, tissue composition, and body site on skin biomechanical properties. Skin research and technology: official journal of International Society for Bioengineering and the Skin 2006; 12: 43-49

2 Calleja-Agius J, Muscat-Baron Y, Brincat MP. Skin ageing. Menopause international 2007; 13: 60-64

3 Proksch E, Schunck M, Zague V et al. Oral intake of specific bioactive collagen peptides reduces skin wrinkles and increases dermal matrix synthesis. Skin pharmacology and physiology 2014; 27: 113-119

4 Proksch E, Segger D, Degwert J et al. Oral supplementation of specific collagen peptides has beneficial effects on human skin physiology: a double-blind, placebo-controlled study. Skin pharmacology and physiology 2014; 27: $47-55$

5 Tanaka M, Koyama Y, Nomura Y. Effects of collagen peptide ingestion on UV-B-induced skin damage. Bioscience, biotechnology, and biochemistry 2009; 73: 930-932

6 Oba C, Ohara H, Morifuji M et al. Collagen hydrolysate intake improves the loss of epidermal barrier function and skin elasticity induced by UVB irradiation in hairless mice. Photodermatology, photoimmunology \& photomedicine 2013; 29: 204-211

7 Ohara $\mathrm{H}$, Matsumoto $\mathrm{H}$, Ito $\mathrm{K}$ et al. Comparison of quantity and structures of hydroxyproline-containing peptides in human blood after 
oral ingestion of gelatin hydrolysates from different sources. Journal of agricultural and food chemistry 2007; 55: $1532-1535$

8 Iwai $K$, Hasegawa T, Taguchi $Y$ et al. Identification of food-derived collagen peptides in human blood after oral ingestion of gelatin hydrolysates. Journal of agricultural and food chemistry 2005; 53: 6531 6536

9 Oesser S, Adam M, Babel W et al. Oral administration of (14)C labeled gelatin hydrolysate leads to an accumulation of radioactivity in cartilage of mice (C57/BL). The Journal of nutrition 1999; 129: 1891 - 1895

10 Bauza E, Oberto G, Berghi A et al. Collagen-like peptide exhibits a remarkable antiwrinkle effect on the skin when topically applied: in vivo study. International journal of tissue reactions 2004; 26: 105111

11 Perrin A, Bauza E, Dal Farra C et al. Stimulating effect of collagen-like peptide on the extracellular matrix of human skin: histological studies. International journal of tissue reactions 2004; 26: 97-104

12 Chai HJ, Li JH, Huang HN et al. Effects of sizes and conformations of fishscale collagen peptides on facial skin qualities and transdermal pene- tration efficiency. Journal of biomedicine \& biotechnology 2010; 2010: 757301

13 Zague $V$. A new view concerning the effects of collagen hydrolysate intake on skin properties. Archives of dermatological research 2008; 300: $479-483$

14 Shimizu J, Asami N, Kataoka A et al. Oral collagen-derived dipeptides, prolyl-hydroxyproline and hydroxyprolyl-glycine, ameliorate skin barrier dysfunction and alter gene expression profiles in the skin. Biochemical and biophysical research communications 2015; 456: 626 630

15 Shigemura Y, Iwai K, Morimatsu F et al. Effect of Prolyl-hydroxyproline (Pro-Hyp), a food-derived collagen peptide in human blood, on growth of fibroblasts from mouse skin. Journal of agricultural and food chemistry 2009; 57: $444-449$

16 Ohara $H$, Ichikawa S, Matsumoto $H$ et al. Collagen-derived dipeptide, proline-hydroxyproline, stimulates cell proliferation and hyaluronic acid synthesis in cultured human dermal fibroblasts. The Journal of dermatology 2010; 37: $330-338$ 